\title{
Impact of barrier tissue inflammation and physical activity on joint
}

\section{homeostasis in mice}

Margot Van Mechelen ${ }^{1,2}$, Tobie Martens ${ }^{3}$, Pieter Vanden Berghe ${ }^{3}$, Rik Lories ${ }^{1,2}$, Giulia R. Gulino $^{1}$

${ }^{1}$ Department of Development and Regeneration, Skeletal Biology and Engineering Research Center, Laboratory of Tissue Homeostasis and Disease, KU Leuven, Leuven, Belgium.

${ }^{2}$ Division of Rheumatology, University Hospitals Leuven, Leuven, Belgium.

${ }^{3}$ Department of Chronic Diseases Metabolism and Ageing, TARGID, Laboratory for Enteric NeuroScience (LENS) and Cell \& tissue Imaging Cluster (CIC), KU Leuven, Leuven, Belgium.

Corresponding author: Dr. Giulia Gulino

Skeletal Biology and Engineering Research Center, O\&N1 - Box 813 - Herestraat 49, B-3000 Leuven, Belgium.

Email: giuliarossana.gulino@kuleuven.be. ORCID ID: 0000-0003-4326-4834.

(C) The Author(s) 2021. Published by Oxford University Press on behalf of the British Society for Rheumatology. All rights reserved. For permissions, please email: journals.permissions@oup.com 


\begin{abstract}
Objectives: To investigate whether physical activity interferes with joint homeostasis in the presence of distant inflammation originating at barrier tissues such as skin or gut.
\end{abstract}

Methods: Eight-week-old male C57/BI6 mice were treated with imiquimod cream on a shaved area of the back skin or with dextran sodium sulphate dissolved in the drinking water to induce psoriasis-like skin or inflammatory bowel disease-like gut inflammation. Afterwards, part of the mice was subjected to a 4-week forced running routine ( $n=10$ per group). Severity of cutaneous or intestinal inflammation was assessed clinically, by histology and by quantitative PCR. Knees and paws were analyzed by micro-computed tomography, histology, immunohistochemistry, second-harmonic generation microscopy and quantitative PCR.

Results: Local induction of inflammation triggered a systemic response with splenomegaly, loss of bone mass and bone marrow changes. Psoriasis- but not inflammatory bowel diseaselike inflammation led to synovial lining layer hyperplasia, an increase in infiltrating CD45+ synovial cells, and suppressed entheseal extracellular matrix gene expression levels. Mechanical loading decreased the amount of F4/80+ synovial macrophages in untreated mice only and led to morphological alterations in the collagen fibers of the enthesis.

Conclusion: Systemic inflammation and mechanical loading act independently of each other. The former, originating from distant sites, can trigger mild synovial inflammation in mice, a propensity that may also impact the development of arthritis in patients; the latter has no impact on the severity of systemic inflammation, but independently affects joint homeostasis. 
Keywords: Spondyloarthritis, psoriasis, inflammatory bowel disease, physical activity

\section{Key messages}

- Imiquimod-, but not dextran sodium sulphate-induced, inflammation induces a mild synovial inflammation in wild-type mice.

- Mechanical loading has no impact on severity of systemic inflammation but independently impacts joint homeostasis.

- Mechanical loading normalizes skin-derived danger signals.

\section{Introduction}

Spondyloarthritis $(\mathrm{SpA})$ is a group of chronic inflammatory joint diseases, which can be classified as either axial or peripheral spondyloarthritis depending on the affected joints, with the prototypes ankylosing spondylitis and psoriatic arthritis respectively [1, 2]. Key features of $\mathrm{SpA}$ are a strong association with the skin disease psoriasis ( $\mathrm{PsO})$ and with inflammatory bowel disease (IBD) [3], and the presence of enthesitis, defined as the inflammation occurring at the insertion of tendons and ligaments into the bone [4], structures critical for locomotion, and sites of biomechanical stress [5].

Increasing evidence suggests that biomechanical stress initiates an inflammatory cascade in the entheses eventually leading to joint disease, possibly involving both stimulation of interleukin-23- or tumor necrosis factor alpha- responsive tissue-resident cells and the recruitment of monocytes [6-8]. Also, the presence of systemic inflammation originating from distant sites has often been considered as a predisposing element for the initiation of SpA. Moreover, evidence from animal models indicates that the type of systemic inflammation 
determines its eventual impact on the joints: overexpression of tumor necrosis factor alpha or interleukin-23 leads to overt arthritis, which is not seen in the case of interleukin-17A overexpression $[7,9,6,10]$.

Here, we describe three mouse models combining PsO-like disease, and acute or chronic IBDlike disease with mechanical loading, and investigate whether the presence of systemic inflammation originating at distant sites and physical activity interferes with joint homeostasis.

\section{Materials and Methods}

\section{Animal experiments}

All animal experiments were approved by the Ethical Committee for Animal Research (KU Leuven, Belgium). Eight weeks old wild-type male C57BI/6 mice were purchased from Janvier (Le Genest-Saint-Isle, France) and maintained under conventional housing conditions.

For the imiquimod-induced PsO model, the back skin of the mice was shaved and subsequently treated with $62.5 \mathrm{mg}$ imiquimod (IMQ) cream (Aldara 5\%, Meda Pharma, Sweden) six days per week for the first week [11], and 3x/week thereafter to maintain chronic skin inflammation. Control mice were shaved and left untreated. After the first week, half of the treated and untreated mice underwent a forced running protocol as previously described [12]. Briefly, mice ran for 4 weeks, 1 hour a day, 5 days a week, at a speed of $11 \mathrm{~m} / \mathrm{min}$ and with an inclination of $5^{\circ}$. We assessed the severity of the skin lesions in the treated animals with the Psoriasis Area and Severity Index (PASI) score, created by the sum of redness, thickness and scaling of the skin, all scored from 0-3. 
For the dextran sodium sulphate-induced colitis model, $2 \%$ dextran sodium sulphate (DSS, 3550kDa, MP Biomedicals, Illkirch, France) was administered through drinking water during 7 days to induce acute colitis, or for 3 cycles of 7 days DSS and 14 days recovery for chronic colitis [13]. Afterwards, half of the treated and untreated mice underwent the same forced running protocol, without further administration of DSS. During the experiment, severity of intestinal inflammation in the treated animals was judged by the Disease Activity Index (DAl), calculated as the sum of body weight loss (one point per $5 \%$ loss of weight), stool consistency $(0$, normal; 2 , soft; 4 , liquid) and presence of gross blood in the stools ( 0 , none; 1 , present).

All experimental groups in each experiment consisted of 10 animals. Our initial experimental set-up was exploratory and no specific power calculation was performed. During the forced running protocol, control mice were kept in the same room to correct for potential confounders. Mice received intraperitoneal injections of $300 \mu \mathrm{l}$ for 4 days in case of excessive weight loss due to inflammation. At the end of each experiment, mice were sacrificed via cervical dislocation. Four mice died prematurely during the experiment and were not included in the analysis. Dot plots indicate the result per animal unless otherwise indicated in the figure legend.

\section{$\underline{\text { Micro CT }}$}

Knees and paws were fixed overnight at $4{ }^{\circ} \mathrm{C}$ in $4 \%$ formaldehyde, followed by storage in PBS at $4^{\circ} \mathrm{C}$ until imaging. To document structural changes in the bone mass, expressed as bone volume/tissue volume (BV/TV), knees were scanned with the ex vivo microCT 1172 (Bruker, Kontich, Belgium), using resolution $5 \mu \mathrm{m}$, source voltage $49 \mathrm{kV}$, source current $200 \mu \mathrm{A}$, filter $0.5 \mathrm{~mm}$, exposure time $590 \mathrm{~ms}$, frame averaging 3 and rotation step $0.3^{\circ}$. Image reconstruction and data analysis were performed with Nrecon and CTan (Bruker). 


\section{Histology}

Eight $\mathrm{mm}$ skin biopsies, part of the colon and the spleens were fixed overnight at $4^{\circ} \mathrm{C}$ in $4 \%$ formaldehyde and embedded in paraffin. After imaging, knees and paws were decalcified for 3 weeks in 0.5 M EDTA pH 7.5 and embedded in paraffin. Hematoxylin-Eosin, HematoxylinSafranin-O staining and immunohistochemistry were performed on $5 \mu \mathrm{m}$-thick sections. Images were acquired with an Olympus IX83 microscope. The adipocyte count per $\mathrm{mm}^{2}$ of bone marrow in the proximal tibia was calculated using Osteomeasure (OsteoMetrics); per mouse, the average value of three different sections is reported. Scoring of the knee joints was performed by three blinded observers on frontal knee sections, taking into account synovial cell infiltration and synovial hyperplasia with each score ranging $0-3$ and assessed on multiple consecutive sections per mouse. For immunohistochemistry, the primary antibodies used were anti-CD45 (eBioscience Thermo Fisher Scientific, 14-0451-81), antiF4/80 (eBioscience Thermo Fisher Scientific, 14-1801-82), anti-MPO (Abcam, ab188211), antiMSCF Receptor (Abcam, ab215441), anti-CD3 (Abcam, ab16669), anti-TLR7 (Thermo Fisher Scientific, PA5-95258), anti-MMP13 (Abcam, ab39012) and anti-IL33 (Abcam, ab187060). Quantification was performed with ImageJ on 40X magnification pictures of two technical replicates for 3 different mice per condition, with normalization to hematoxylin staining intensity and to the average of the untreated control group. Tendon thickness was measured in ImageJ, averaging measurements at three different levels in the tendon in a $90^{\circ}$ angle with the fiber direction.

\section{RNA extraction}


Parts of the skin and colon were snap-frozen and homogenized in $\beta$-mercapto-ethanol using the Precellys 24 tissue homogenizer (Bertin), followed by RNA isolation using the Nucleospin RNA mini kit (Machery-Nagel). Achilles tendons including the enthesis were snap-frozen and homogenized in Trizol (Invitrogen), followed by RNA isolation using a manual phenol/chloroform extraction protocol. RNA from previously fixed tendons was isolated using the PureLink FFPE RNA Isolation Kit (Invitrogen). cDNA was synthesized with the RevertAid H Minus First Strand cDNA Synthesis Kit (Fermentas) according to the manufacturer's recommendations. Real-time RT-PCR was performed on a Corbett Rotor-gene qPCR machine (Qiagen) using Maxima SYBR Green qPCR Master Mix (Fermentas), or TaqMan Fast Advanced Master Mix (Applied Biosystems) in case of previous fixation. Relative quantification was obtained with the $\Delta \Delta \mathrm{Ct}$ method, using Gapdh as internal control for skin and colon, and $T b p$ and $B 2 \mathrm{~m}$ for tendon tissue. In skin and colon, II33, Cxcl1, Cc/2,Ccl20, Ido1 and Zo-1 were detected, with the addition of Reg3g for colon samples. Col1a1, Col3a1, and Tgfb were detected in the tendons.

\section{2-photon autofluorescence (2PAF) and second harmonic generation (SHG) imaging}

Achilles tendon images were acquired of $5 \mu \mathrm{m}$ thick intact hind paw sections on a LSM 780 confocal microscope (Zeiss) using a $25 \mathrm{X}$ water immersion objective (0.8 NA, Zeiss). Samples were excited with a tunable Mai Tai DeepSee Titanium-Sapphire pulsed femtosecond laser (680-1050 nm; Spectra-Physics) set at $850 \mathrm{~nm}$ for simultaneous 2PAF and SHG imaging. BiG (GaAsP) non-descanned detectors (Zeiss) were used for recording both 2PAF and SHG signals. 2PAF signals were captured in epi configuration (emission filter: $500-550 \mathrm{~nm}$ ) and SHG signals were captured in forward (emission filter: $380-430 \mathrm{~nm}$ ) direction. Images were adjusted for brightness and contrast. Fiber extraction and quantification was performed using CT-FIRE [14]. 


\section{$\underline{\text { Statistical analysis }}$}

All statistical analyses were performed using GraphPad Prism 7. Assessment of normality of the data was evaluated by histogram presentation of the data and QQ plots of the residuals. When necessary, a logarithmic transformation was applied. Gene expression data were logarithmic transformed. For the PASI and DAI scores, a 2-way repeated measurements ANOVA was used, or a mixed-effect analysis when values were missing. All group comparisons thereafter were performed using a two-way ANOVA. A p-value $\leq 0.05$ was considered statistically significant. An overview of the results can be found in Supplementary Data S1, available at Rheumatology online.

\section{Results \\ $\underline{\text { Induction of local inflammation leads to a systemic response }}$}

Local inflammation in mice was induced by IMQ or DSS and followed by a 4-week forced running protocol. IMQ-treated mice developed PsO-like skin as indicated by the PASI score (Fig. 1A), with acanthosis, hyperkeratosis and a cellular infiltrate as highlighted by pathology analysis (Fig. 1B). DSS-treated mice developed colitis with weight loss, diarrhea and bloody stools as indicated by the DAI-score (Fig. 1A), with a cellular infiltrate and altered mucosal architecture on histology (Fig. 1B).

A systemic response was first highlighted by the evident splenomegaly in the IMQ-, but not DSS-, treated mice (Fig. 1C). In both IMQ- and DSS-treated mice, there was a loss of bone mass (Fig. 1D) [difference of the means $8.68 \%(95 \% \mathrm{Cl}$ : 6.72 - 10.64) with $\mathrm{p}<0.001$ for $\mathrm{IMQ}, 4.47 \%$ $(95 \% \mathrm{Cl}: 2.09-6.85)$ with $\mathrm{p}=0.005$ for acute DSS, $4.77 \%(95 \% \mathrm{Cl}: 2.82-6.60)$ with $\mathrm{p}<0.0001$ for chronic DSS] and a decrease in the number of bone marrow adipocytes [difference of the 
means $93.49 / \mathrm{mm}^{2}(95 \% \mathrm{Cl}: 58.39$ - 128.6) with $\mathrm{p}<0.0001$ for IMQ, 92.12/mm² (95\%Cl: 62.32 - 121.9) with $p<0.0001$ for acute DSS, $32.10 / \mathrm{mm}^{2}(95 \% \mathrm{Cl}: 10.97-53.23)$ with $\mathrm{p}=0.0053$ for chronic DSS] (Fig. 1E). Forced exercise did not exert any effect on these parameters and no interaction between the two variables was found in the analysis.

\section{$\underline{\text { Physical activity reduces skin-, but not intestinal-, derived danger signals }}$}

Disease severity during the experiment and on end-stage histology of skin and intestine did not differ between non-running and running groups in any of the models (Fig. $1 \mathrm{~A}$ and B). Despite this, in the skin of IMQ-treated mice gene expression and protein levels of alarmin I/33 normalized with running (Fig. 2A) [difference of the means 4.09 fold $(95 \% \mathrm{Cl}: 1.86-8.97)$ with $p=0.0002$ for IMQ non-running vs running], as did gene expression levels of chemokines Cxcl1 [difference of the means 9.06 fold (95\% Cl: $2.92-28.18)$ with $p<0.0001$ for IMQ nonrunning vs running] and $C c / 20$ [difference of the means 4.62 fold $(95 \% \mathrm{Cl}: 1.2-17.78)$ with $\mathrm{p}=$ 0.0213 for IMQ non-running vs running], but not $C C / 2$ (Fig. 2B). However, the number of neutrophils in the skin remained unaltered as judged by immunohistochemistry for MPO (data not shown). In the intestine of the DSS models, no changes in chemokine expression were detected upon running (Suppl. Fig. S1A, available at Rheumatology online.). Besides leukocyte recruitment, we found no evidence that other common barrier functions such as microbial sensing and barrier integrity were altered by physical activity, with stable expression levels of Ido1 and Reg3g, and Zo-1 respectively (Suppl. Fig. S1B, available at Rheumatology online.).

\section{$\underline{\text { PsO- but not IBD-like inflammation affects the cellular composition of the synovium }}$}

No clinical signs of joint disease were detected in the animals during the course of the experiments. However, histological examination of the knees showed mild hyperplasia of the synovial lining layer [difference of the means 0.35 units $(95 \% \mathrm{Cl}: 0.10-0.60)$ with $\mathrm{p}=0.0072$ 
for IMQ vs control] and increased cellular infiltration in all IMQ-, but not DSS-treated mice compared to control mice [difference of the means 0.54 units $(95 \% \mathrm{Cl}: 0.25-0.82)$ with $\mathrm{p}=$ 0.0005 for IMQ vs control] (Fig. 3A and data not shown). Cells responsible for the cellular infiltrate in the IMQ-treated mice were identified as CD45+ cells. The analysis showed that the number of CD45+ cells (Fig. 3B) was impacted by both IMQ treatment and exercise. IMQ treatment increased the immunoreactive signal in sections from non-running [difference of the means 209.4 fold $(95 \% \mathrm{Cl}: 105.0-418.6)$ with $\mathrm{p}<0.0001$ ] as well as running mice [difference of the means 12.39 fold $(95 \% \mathrm{Cl}: 6.21-24.75)$ with $\mathrm{p}<0.0001$ ]. Running increased the number of CD45+ cells in non-IMQ exposed mice [difference of the means 11 fold $(95 \% \mathrm{Cl}$ : $5.51-21.96$ ) with $p<0.0001$ ] but no additional effect of running was noted when comparing running and non-running IMQ mice. When further investigating the nature of the CD45+ cells, IMQ treatment increased the immunoreactive signal for macrophage surface marker $\mathrm{F} 4 / 80$ in running [difference of the means 6.06 fold $(95 \% \mathrm{Cl}: 2.81-13.07)$ with $p=0.0003$ ] but not nonrunning mice [difference of the means 1.84 fold $(95 \% \mathrm{Cl}: 1.17-3.97)$ with $p=0.1263$ ]. In the untreated mice on the other hand, physical activity decreased the amount of F4/80+ cells [difference of the means 5.10 fold $(95 \% \mathrm{Cl}: 2.36$ to 11.01$)$ with $p=0.0006$ for control nonrunning vs control running] (Fig. 3C). There was no difference in the amount of MCSF-R+ or TLR7+ cells between the groups, and no MPO+ or CD3+ cells were detected (data not shown).

\section{Systemic inflammation does not affect the cartilage}

Treatment with IMQ or DSS, with or without forced exercise, did not induce structural cartilage damage, proteoglycan loss or ectopic hypertrophy of chondrocytes in the knee articular cartilage as judged by Safranin-O staining and immunohistochemistry for MMP13 (Suppl. Fig. S2, available at Rheumatology online.). 


\section{$\underline{\text { PsO- but not IBD-like inflammation suppresses entheseal extracellular matrix gene expression }}$}

Histological analysis of the Achilles tendons' synovio-entheseal complex did not reveal the presence of cellular infiltrate, but in 2 out of 10 mice of the IMQ running group entheseophytes developed from the cuboidal bone, as compared to none in the other groups (Fig. 4A). In the Achilles tendons of IMQ-, but not DSS-treated mice, there was reduced gene expression of collagen 1a1 (Col1a1) [difference of the means 2.40 fold (95\% $\mathrm{Cl}$ : $1.11-5.29)$ with $\mathrm{p}=0.0295$ for control vs IMQ] and 3a1 (Col3a1) [difference of the means 4.09 fold $(95 \% \mathrm{Cl}$ : $1.86-8.97)$ with $p<0.0001$ for control vs IMQ], accompanied by an increase in the levels of transforming growth factor $\beta$ expression (Tgfb) [difference of the means 2.08 fold $(95 \% \mathrm{Cl}: 1.47$ -2.95) with $p=0.0004$ for IMQ vs control], with no influence of the forced exercise (Fig. 4B). On the contrary, the running regimen led to an increased tendon thickness in untreated mice only and an increased amount of collagen type I fibers regardless of the treatment. The width and length distribution of the fibers remained unchanged, though an increased number of fibers was recorded for the same intervals (Fig. 4C and D).

\section{Discussion}

SpA is a chronic inflammatory musculoskeletal disease in which skin and gut inflammation, and mechanical stress have been hypothesized as triggers for onset of joint disease, but experimental evidence to support these concepts is still limited $[3,5]$.

The association between SpA and skin or gut inflammation $[15,16]$, is a connection reflected in multiple transgenic animal models mimicking human SpA [17]. The direct impact of systemic 
inflammation on the joints largely depends on the type of inflammation: while systemic overexpression of tumor necrosis factor alpha leads to erosive arthritis and enthesitis, overexpression of interleukin-23 by injecting minicircle DNA induces a more SpA-like phenotype with both erosions and new bone formation $[6,7]$. On the contrary, interleukin$17 \mathrm{~A}$, either by injection of minicircle DNA or originating from the skin in PsO mouse models $[10,6]$, only elicits bone loss without concomitant arthritis. Here we demonstrate that both IMQ-induced PsO- and DSS-induced IBD-like inflammation lead to bone loss. Additionally, IMQ, but not DSS treatment, induces mild synovial and entheseal changes in the peripheral joints, even in the absence of a susceptible genetic background.

Likewise, biomechanical stress is highly associated with SpA [5]. Previous reports have identified mechanical loading as an important factor in both onset and chronicity of inflammation in several widely used animal models of peripheral arthritis, all having a background of systemic inflammation $[7,8,18]$. In contrast, the applied biomechanical stress in our models of induced PsO- or IBD-like disease did not induce arthritis, but did lead to adaptive changes in both the synovium and entheses. In general, exercise is considered to be beneficial in health and disease, including those characterized by inflammation [19]. In spondyloarthritis, the role of exercise is generally seen as positive but type of exercise and associated strain are debated as mechanical strain and damage have been linked to disease development at certain sites [20-22]. In our set-up, we did not observe specific effects of exercise on the severity of disease in the IMQ and DSS models, despite the reduction in some danger signals in the IMQ model. It has been shown earlier that physical activity does not impact the severity of systemic inflammation [18], however, our study was not designed to address this issue and the exercise protocol not developed for studying eventual beneficiary effects. 
Remarkably, the impact of distant inflammation seen here developed irrespective of the presence of increased mechanical loading and vice versa, indicating that these factors may act independently of each other on the joint.

The limitations of this study include the absence of direct comparison of the severity of systemic inflammation between the PsO- or IBD-like inflammation. Although systemic inflammation appears to be triggered by the local exposure to both immune-stimulating agents, no direct comparison of the systemic effects is possible due to the different underlying mechanisms of immune activation and the distinct anatomical site where inflammation is triggered. Secondly, we cannot rule out higher systemic effects due to the absorption of IMQ through the skin because of its low molecular weight in contrast to the large DSS molecules, and although the extensive local exposure of the skin to IMQ is considered the main factor triggering both local and systemic inflammation in our model, ingestion of small amounts of IMQ by the animals cannot be excluded as a factor that contributes to the effects observed [23]. Therefore, the downstream phenotypes in our study may be linked to the stimulus rather than the site of inflammation.

In addition, the use of locally induced PsO- or IBD-like disease does not take into account the importance of a susceptible genetic background [24], but this experimental set-up enables the unbiased interpretation of the effects of barrier tissue derived inflammation and mechanical loading, limiting the interference of unknown variables. Finally, another limitation of this study is the running regimen that was forced upon the animals. We have chosen a well-tolerated protocol based on our earlier experience but cannot exclude that more strenuous exercise would have had different effects on the joint. Yet, in our set-up we needed to take into account the severity of the IMQ and DSS models that in our view would limit their performance 
in ad libitum running or might result in excessive suffering of the animals in more intensive and extensive protocols.

In summary, our findings provide evidence that barrier tissue inflammation and biomechanical loading can influence joint homeostasis independently from each other. Further research is necessary to investigate whether this altered joint homeostasis can be responsible for a different threshold of the joints to respond to damaging stimuli eventually leading to joint disease. 


\section{Acknowledgements}

The authors thank Tine Peeters for contributing to scoring the histopathology sections. Tobie Martens, staff scientist at the KU Leuven Core facility, Cell and tissue Imaging Cluster (CIC), recorded and analyzed the autofluorescence and SHG images on a Zeiss LSM 780 - SP Mai Tai HP DS.

\section{Funding}

This work is supported by the Flanders Research Foundation (FWO-Vlaanderen) [grant numbers G094614 to Margot Van Mechelen and G095916N to Giulia R. Gulino] and Group for Research and Assessment of Psoriasis and Psoriatic Arthritis (GRAPPA) [pilot research grant to Margot Van Mechelen]. The 2-photon autofluorescence and second harmonic generation imaging was supported by Hercules [grant number AKUL/11/37 to Pieter Vanden Berghe] and FWO-Vlaanderen [grant number G092915 to Pieter Vanden Berghe].

\section{Disclosure statement}

Leuven Research and Development, the technology transfer office of KU Leuven has received consultancy and speaker fees, and research grants on behalf of Rik Lories from Abbvie, Amgen (Celgene), Boehringer-Ingelheim, Eli-Lilly, Galapagos, Janssen, Kabi-Fresenius, MSD, Novartis, Pfizer, Sandoz, Samumed and UCB.

\section{Data availability statement}

The data that support the findings of this article will be shared on reasonable request to the corresponding author. 


\section{References}

1. Sieper J, Poddubnyy D. Axial spondyloarthritis. Lancet. 2017. doi:10.1016/S0140-6736(16)31591-4.

2. Ritchlin CT, Colbert RA, Gladman DD. Psoriatic Arthritis. N Engl J Med. 2017;376(21):2095-6. doi:10.1056/NEJMc1704342.

3. Haroon M, FitzGerald O. Psoriatic arthritis: complexities, comorbidities and implications for the clinic. Expert Rev Clin Immunol. 2016;12(4):405-16. doi:10.1586/1744666X.2016.1139453.

4. Schett G, Lories RJ, D'Agostino MA, Elewaut D, Kirkham B, Soriano ER et al. Enthesitis: from pathophysiology to treatment. Nat Rev Rheumatol. 2017;13(12):731-41. doi:10.1038/nrrheum.2017.188.

5. Gracey E, Burssens A, Cambre I, Schett G, Lories R, McInnes IB et al. Tendon and ligament mechanical loading in the pathogenesis of inflammatory arthritis. Nat Rev Rheumatol. 2020;16(4):193-207. doi:10.1038/s41584-019-0364-x.

6. Sherlock JP, Joyce-Shaikh B, Turner SP, Chao CC, Sathe M, Grein J et al. IL-23 induces spondyloarthropathy by acting on ROR-gammat+ CD3+CD4-CD8- entheseal resident T cells. Nat Med. 2012;18(7):1069-76. doi:10.1038/nm.2817.

7. Jacques $P$, Lambrecht $S$, Verheugen E, Pauwels E, Kollias G, Armaka $M$ et al. Proof of concept: enthesitis and new bone formation in spondyloarthritis are driven by mechanical strain and stromal cells. Ann Rheum Dis. 2014;73(2):437-45. doi:10.1136/annrheumdis-2013-203643.

8. Cambre I, Gaublomme D, Burssens A, Jacques P, Schryvers N, De Muynck A et al. Mechanical strain determines the site-specific localization of inflammation and tissue damage in arthritis. Nat Commun. 2018;9(1):4613. doi:10.1038/s41467-018-06933-4.

9. Kontoyiannis D, Pasparakis M, Pizarro TT, Cominelli F, Kollias G. Impaired on/off regulation of TNF biosynthesis in mice lacking TNF AU-rich elements: implications for joint and gut-associated immunopathologies. Immunity. 1999;10(3):387-98. doi:10.1016/s1074-7613(00)80038-2.

10. Uluckan O, Jimenez M, Karbach S, Jeschke A, Grana O, Keller J et al. Chronic skin inflammation leads to bone loss by IL-17-mediated inhibition of Wnt signaling in osteoblasts. Sci Transl Med. 2016;8(330):330ra37. doi:10.1126/scitranslmed.aad8996.

11. van der Fits L, Mourits S, Voerman JS, Kant M, Boon L, Laman JD et al. Imiquimod-induced psoriasislike skin inflammation in mice is mediated via the IL-23/IL-17 axis. J Immunol. 2009;182(9):5836-45. doi:10.4049/jimmunol.0802999.

12. Bomer N, Cornelis FM, Ramos YF, den Hollander W, Storms L, van der Breggen R et al. The effect of forced exercise on knee joints in Dio2(-/-) mice: type II iodothyronine deiodinase-deficient mice are less prone to develop OA-like cartilage damage upon excessive mechanical stress. Ann Rheum Dis. 2016;75(3):571-7. doi:10.1136/annrheumdis-2014-206608.

13. Breynaert C, Dresselaers T, Perrier C, Arijs I, Cremer J, Van Lommel L et al. Unique gene expression and MR T2 relaxometry patterns define chronic murine dextran sodium sulphate colitis as a model for connective tissue changes in human Crohn's disease. PLoS One. 2013;8(7):e68876. doi:10.1371/journal.pone.0068876.

14. Bredfeldt JS, Liu Y, Pehlke CA, Conklin MW, Szulczewski JM, Inman DR et al. Computational segmentation of collagen fibers from second-harmonic generation images of breast cancer. J Biomed Opt. 2014;19(1):16007. doi:10.1117/1.JBO.19.1.016007.

15. Jacques $P$, Elewaut $D$, Mielants $H$. Interactions between gut inflammation and arthritis/spondylitis. Curr Opin Rheumatol. 2010;22(4):368-74. doi:10.1097/BOR.0b013e3283393807.

16. Neerinckx B, Elewaut D, Lories RJ. Spreading spondyloarthritis: are ILCs cytokine shuttles from base camp gut? Ann Rheum Dis. 2015;74(9):1633-5. doi:10.1136/annrheumdis-2015-207735.

17. Vieira-Sousa E, van Duivenvoorde LM, Fonseca JE, Lories RJ, Baeten DL. Review: animal models as a tool to dissect pivotal pathways driving spondyloarthritis. Arthritis Rheumatol. 2015;67(11):2813-27. doi:10.1002/art.39282. 
18. Cambre I, Gaublomme D, Schryvers N, Lambrecht S, Lories R, Venken K et al. Running promotes chronicity of arthritis by local modulation of complement activators and impairing $\mathrm{T}$ regulatory feedback loops. Ann Rheum Dis. 2019;78(6):787-95. doi:10.1136/annrheumdis-2018-214627.

19. Pedersen BK. The Physiology of Optimizing Health with a Focus on Exercise as Medicine. Annu Rev Physiol. 2019;81:607-27. doi:10.1146/annurev-physiol-020518-114339.

20. Debusschere K, Cambre I, Gracey E, Elewaut D. Born to run: The paradox of biomechanical force in spondyloarthritis from an evolutionary perspective. Best Pract Res Clin Rheumatol. 2017;31(6):887-94. doi:10.1016/j.berh.2018.07.011.

21. Van Mechelen M, Lories R. Spondyloarthritis on the Move: Biomechanical Benefits or Harm. Curr Rheumatol Rep. 2020;22(8):35. doi:10.1007/s11926-020-00913-8.

22. Perrotta FM, Lories R, Lubrano E. To move or not to move: the paradoxical effect of physical exercise in axial spondyloarthritis. RMD Open. 2021;7(1). doi:10.1136/rmdopen-2020-001480.

23. Grine L, Steeland S, Van Ryckeghem S, Ballegeer M, Lienenklaus S, Weiss S et al. Topical imiquimod yields systemic effects due to unintended oral uptake. Sci Rep. 2016;6:20134. doi:10.1038/srep20134. 24. Gracey E, Vereecke L, McGovern D, Frohling M, Schett G, Danese S et al. Revisiting the gut-joint axis: links between gut inflammation and spondyloarthritis. Nat Rev Rheumatol. 2020;16(8):415-33. doi:10.1038/s41584-020-0454-9.

Figure legends 
Figure 1: Systemic response to local inflammation. (A) Psoriasis area severity index (PASI) and disease activity index (DAI) scores of imiquimod (IMQ)- and acute and chronic dextran sodium sulphate (DSS)-treated mice (mean $+\mathrm{SE}, \mathrm{n}=8-10$ per group). (B) Representative hematoxylin and eosin staining of skin and colon. (C) Representative picture of the spleens in control (left) and IMQ-treated mice (right). (D) \% ratio of bone volume to tissue volume (BV/TV) of trabecular bone in the tibial epiphysis. Data points represent the average value of the left and right knees (mean $+\mathrm{SD})$. (E) Adipocyte count per $\mathrm{mm}^{2}$ of bone marrow (mean $+\mathrm{SD}$ ). Inserts represent examples for high and low count adipocytes. All scale bars depict $100 \mu \mathrm{m}$. Two-way ANOVA, $* * * p \leq 0.001$

Figure 2: Effect of mechanical loading on danger signals from skin-derived inflammation. (A) Gene expression levels of interleukin 33 (//33) in the skin of control (Ctrl) and imiquimod (IMQ)-treated mice with or without forced running by quantitative real-time PCR analysis (mean \pm SD). Inserts show representative pictures of immunohistochemistry with anti-IL33, performed in 3 animals per group, scale bar $50 \mu \mathrm{m}$. (B) Gene expression levels of $C x c / 1, C c / 20$ and $C C / 2$ in the skin by quantitative real-time PCR analysis (mean $\pm S D$ ). Two-way ANOVA *** $p \leq 0.001, * p \leq 0.5$

Figure 3: Synovial response to inflammation and biomechanical stress in the IMQ model. (A) Histological score of synovial hyperplasia and cellular infiltrate in the knee joints of control (Ctrl) and imiquimod (IMQ)-treated mice with or without forced running. Score ranges $0-3$ (mean \pm SD). Inserts represent examples of high and low scores for hyperplasia and cellular infiltration. (B) Immunohistochemical detection of CD45 in knee synovium with corresponding quantification (mean \pm SD). (C) Immunohistochemical detection of F4/80 in 
knee synovium with corresponding quantification (mean \pm SD). All scale bars depict $100 \mu \mathrm{m}$. Two-way ANOVA *** $p \leq 0.001, * * p \leq 0.01$

Figure 4: Entheseal response to inflammation and biomechanical stress in the IMQ model.

(A) Enthesophyte formation at the cuboidal bone in an imiquimod (IMQ) forced-to-run mice (arrow). (B) Quantitative real-time PCR analysis of collagen 1a1 (Col1a1), collagen 3a1 (Col3a1) and transforming growth factor $\beta$ (Tgfb) in the Achilles tendons (mean + SD). (C) Two-photon autofluorescence (2PAF, red) and SHG (green) pictures of the Achilles tendon insertion with quantification of the tendon thickness and analysis of the number of fibers. (D) Width and length distribution of the fibers as analyzed in CT-FIRE. All scale bars depict $100 \mu \mathrm{m}$. Two-way ANOVA $* * * p \leq 0.001, * p \leq 0.5$ 
Figure 1
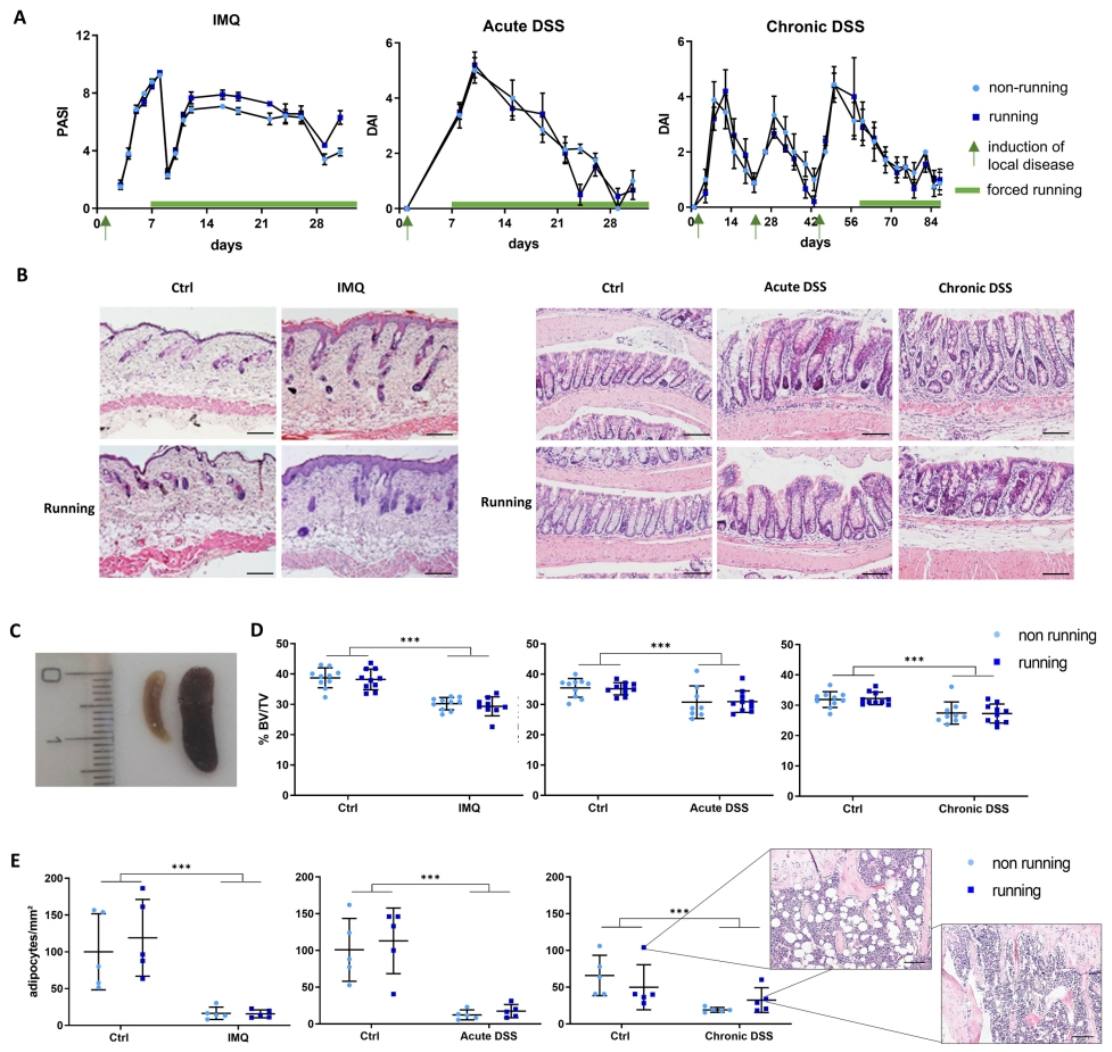

$209 \times 297 \mathrm{~mm}(300 \times 300 \mathrm{DPI})$ 
Page 21 of 31

Rheumatology

$$
\begin{aligned}
& 1 \\
& 2 \\
& 3 \\
& 4 \\
& 5 \\
& 6 \\
& 7 \\
& 8 \\
& 9 \\
& 10 \\
& 11 \\
& 12 \\
& 13 \\
& 14 \\
& 15 \\
& 16 \\
& 17 \\
& 18 \\
& 19 \\
& 20 \\
& 21 \\
& 22 \\
& 23 \\
& 24 \\
& 25 \\
& 26 \\
& 27 \\
& 28 \\
& 29 \\
& 30 \\
& 31 \\
& 32 \\
& 33 \\
& 34 \\
& 35 \\
& 36 \\
& 37 \\
& 38 \\
& 39 \\
& 40 \\
& 41 \\
& 42 \\
& 43 \\
& 44 \\
& 45 \\
& 46 \\
& 47 \\
& 48 \\
& 49 \\
& 50 \\
& 51 \\
& 52 \\
& 53 \\
& 54 \\
& 55 \\
& 56 \\
& 57 \\
& 58 \\
& 59 \\
& 60
\end{aligned}
$$

Figure 2

A

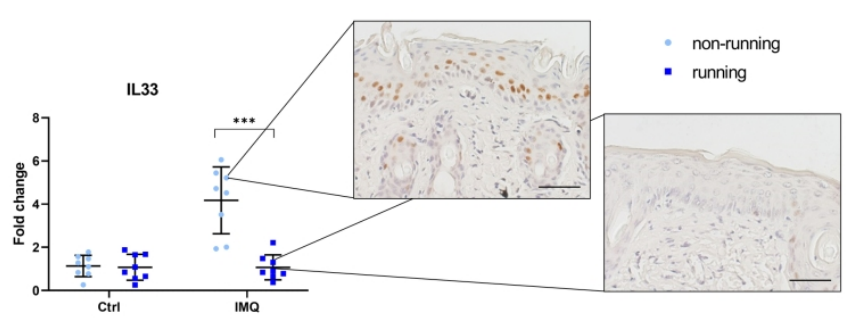

B

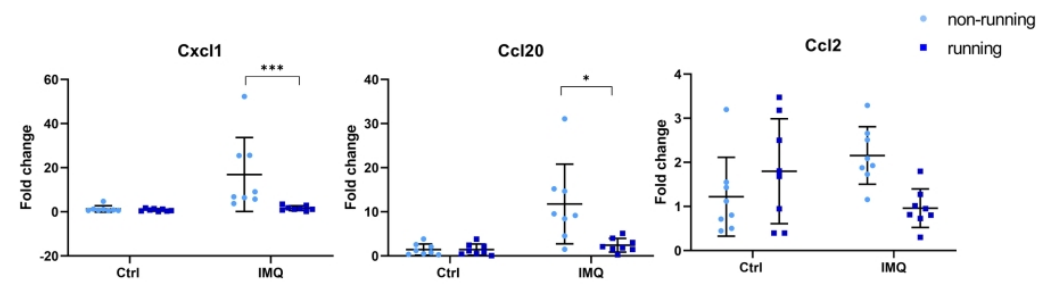

209×297mm (300 x 300 DPI)

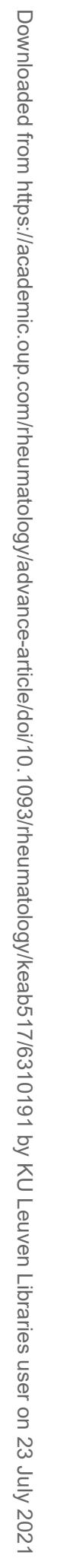


Figure 3

A
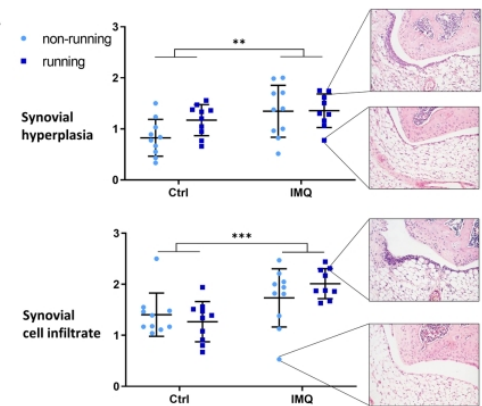

c

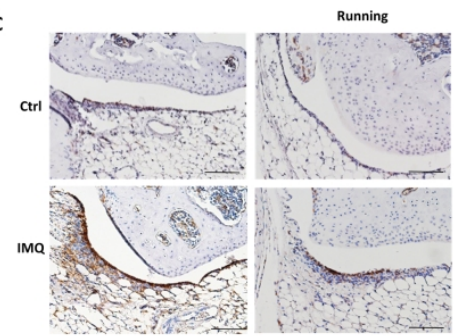

B
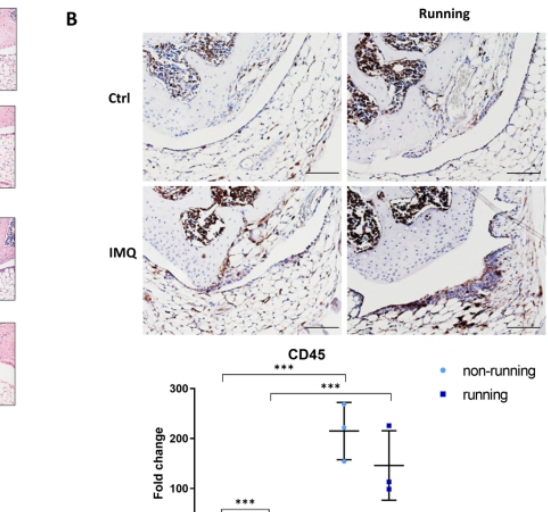

$209 \times 297 \mathrm{~mm}(300 \times 300 \mathrm{DPI})$

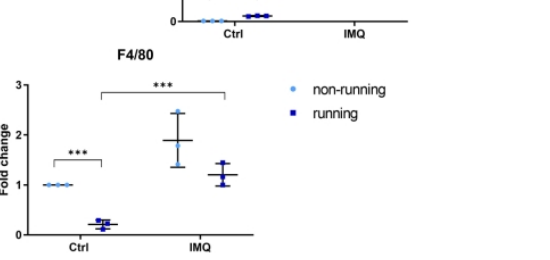




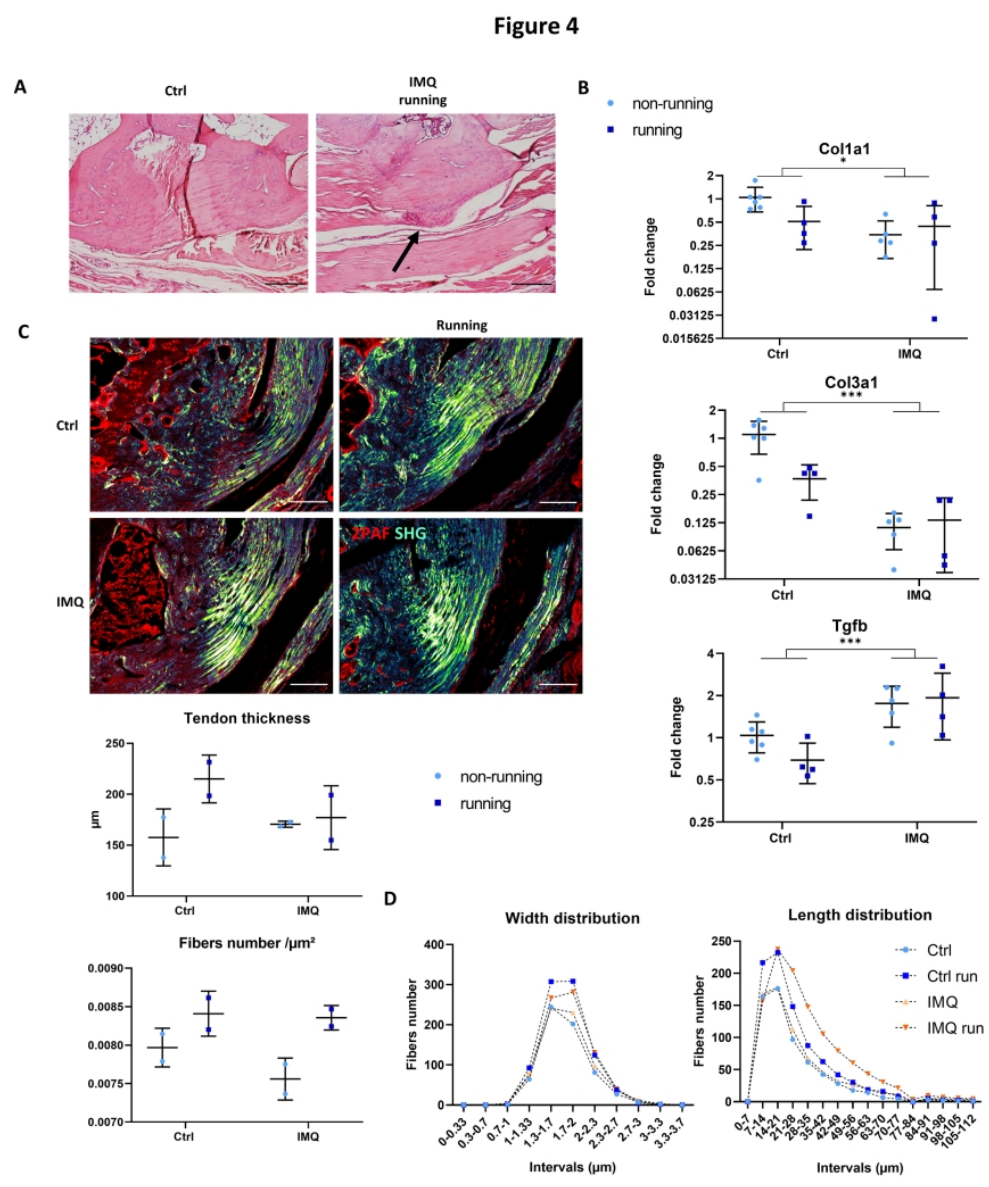

$209 \times 297 \mathrm{~mm}(300 \times 300 \mathrm{DPI})$ 


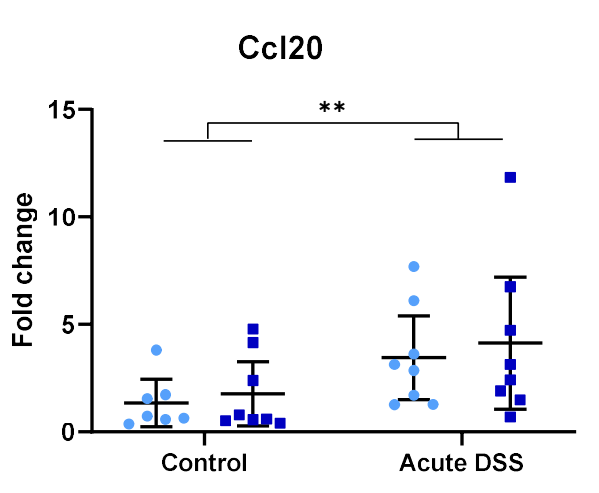

- non-running
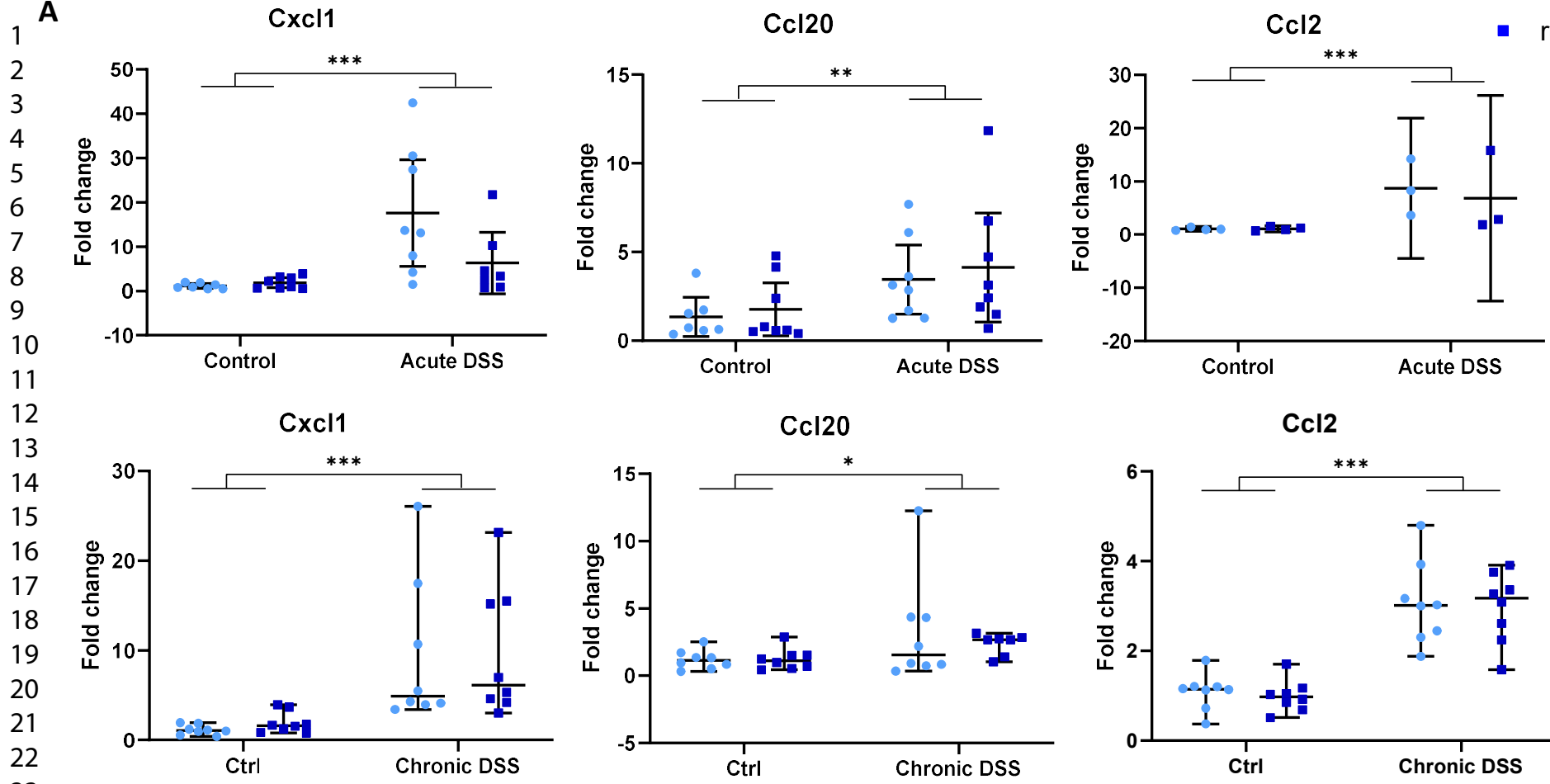

running
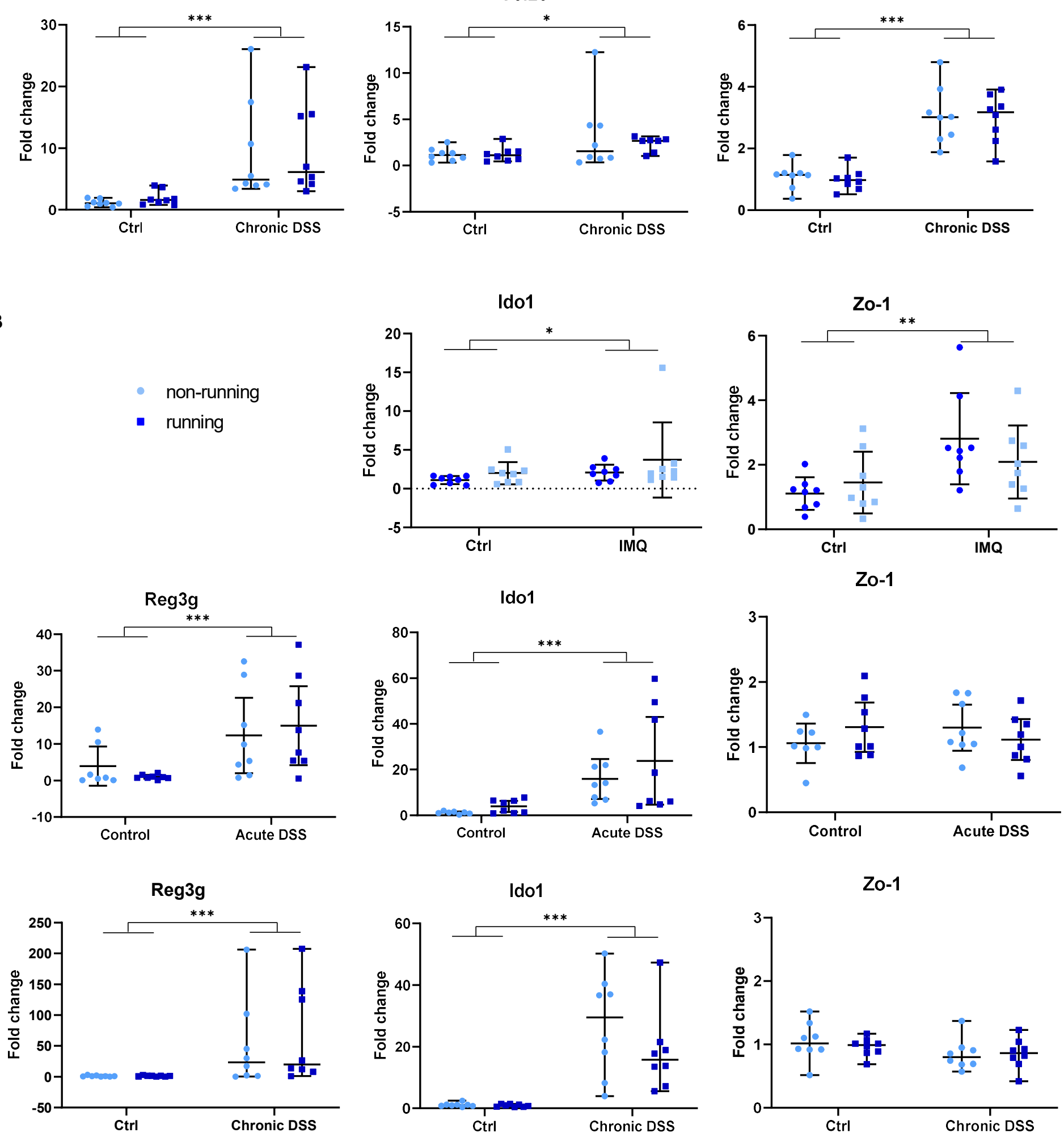
Supplementary Figure S1: Effect of mechanical loading on local cutaneous or intestinal inflammation. (A) Colonic gene expression levels of $\mathrm{Cxcl} 1, \mathrm{Ccl} 20$ and $\mathrm{Ccl} 2$ in control (Ctrl) and dextran sodium sulphate (DSS)treated mice with or without forced running by quantitative real-time PCR analysis (mean \pm SD). (B) Gene expression levels of Ido1, Zo-1 and Reg3g in skin or colon in Ctrl and imiquimod (IMQ)-treated mice with or without forced running by quantitative real-time PCR analysis (mean \pm SD). ${ }^{* * *} p \leq 0.001, * * p \leq 0.01, * p \leq$ 0.5 . 


\section{Suppennfigilsgy}

$1 \mathrm{~A}$

2

3

4

5

6

7

8

9

10

11

Rtynning

13

14

15

$17^{B}$

18

19

20

21

22

23

24

25

26

27

28

29

30

Ŗunning

32

33

34

35

36

37

38

39

40

41

42

43

44

45

46

47

48

49

50

51

52

53

54

55

56

57

58

59

60
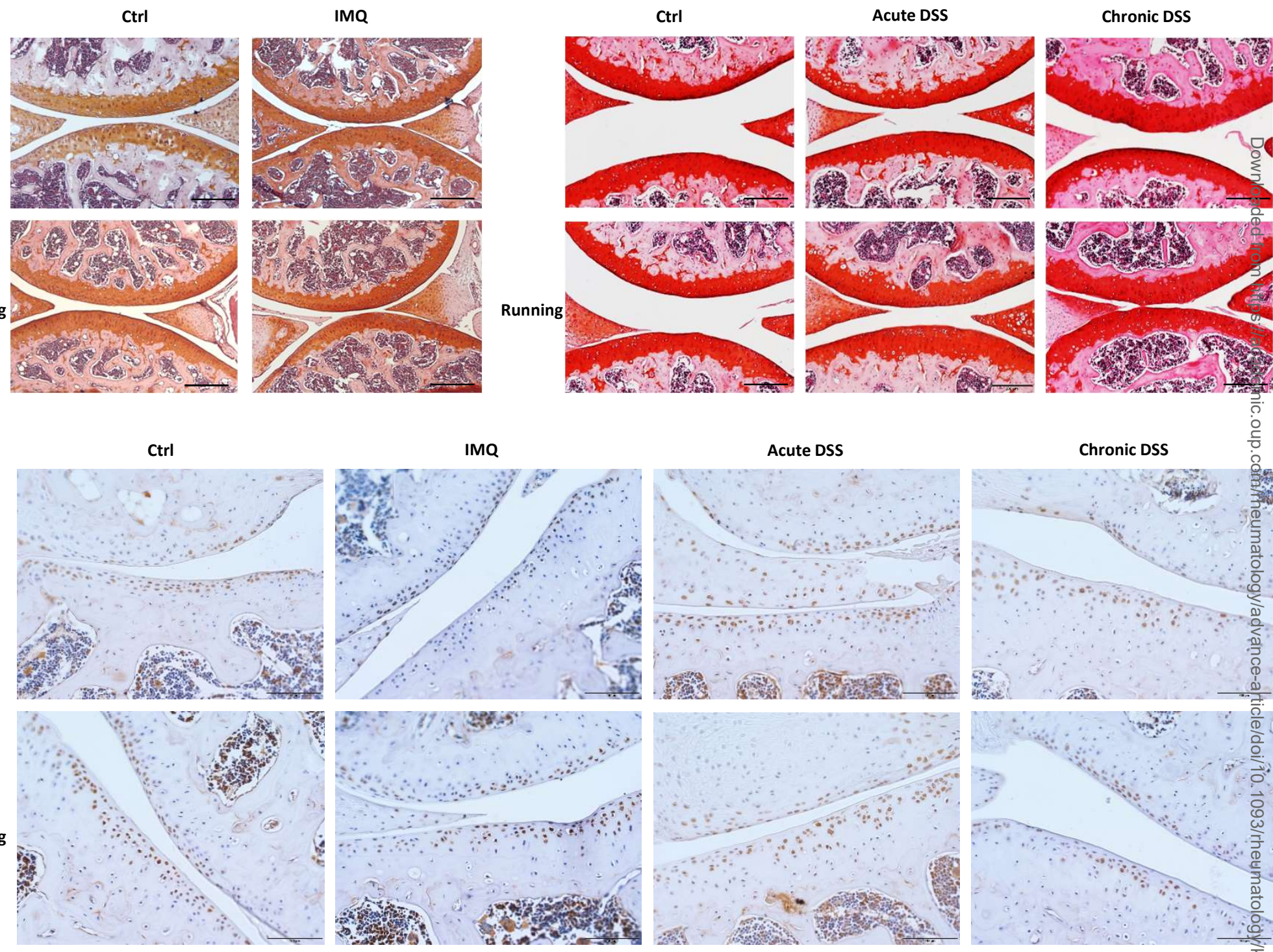
Supplementary Figure S2: Evaluation of the effect of mechanical loading and systemic inflammation on the cartilage. (A) Representative hematoxylin and Safranin-O stained knee sections in the IMQ and both DSS models. Scale bars $200 \mu \mathrm{m}$. (B) Representative pictures of immunohistochemical detection of MMP13 in the knee cartilage in the IMQ and both DSS models. Scale bars $100 \mu \mathrm{m}$. 


\section{Supplementary Data S1. Overview statistical results}

Fig.1D: \% ratio of bone volume to tissue volume (BV/TV)

IMQ:

difference of the means Ctrl vs IMQ 8.68\% (95\%Cl: 6.72 - 10.64)

$\begin{array}{llll}\text { main effect IMQ } & F(1,35)=80.96 & p<0.001 \quad \text { (two-way ANOVA) }\end{array}$

Acute DSS:

difference of the means Ctrl vs DSS $4.47 \%$ (95\%Cl: 2.09 - 6.85)

$\begin{array}{llll}\text { main effect DSS } & F(1,34)=14.57 \quad p=0.005 \quad \text { (two-way ANOVA) }\end{array}$

Chronic DSS:

difference of the means Ctrl vs DSS 4.77\% (95\%Cl: 2.82 - 6.60)

main effect DSS $\quad F(1,35)=25.64 \quad p<0.0001 \quad$ (two-way ANOVA)

Fig.1E: Adipocyte count per $\mathrm{mm}^{2}$ of bone marrow

IMQ:

difference of the means Ctrl vs IMQ $93.49 / \mathrm{mm}^{2}(95 \% \mathrm{Cl}: 58.39$ - 128.6)

$\begin{array}{llll}\text { main effect IMQ } & F(1,16)=31.88 \quad p<0.0001 \quad \text { (two-way ANOVA) }\end{array}$

Acute DSS:

difference of the means Ctrl vs DSS $92.12 / \mathrm{mm}^{2}$ (95\% Cl: 62.32 - 121.9)

main effect DSS $\quad F(1,16)=42.94 \quad p<0.0001 \quad$ (two-way ANOVA)

Chronic DSS:

difference of the means Ctrl vs DSS $32.10 / \mathrm{mm}^{2}$ (95\%Cl: $\left.10.97-53.23\right)$

$\begin{array}{llll}\text { main effect DSS } & F(1,16)=10.37 \quad p=0.0053 \quad \text { (two-way ANOVA) }\end{array}$ 
Fig.2A: Gene expression level of interleukin 33 (I/33) in the skin

difference of the means IMQ running vs IMQ non-running 4.09 fold $(95 \% \mathrm{CI}: 1.86-8.97)$

interaction $\quad F(1,28)=10.34 \quad p=0.0033 \quad$ (two-way ANOVA)

IMQ running vs IMQ non-running $\quad \mathrm{p}=0.0002 \quad$ (Tukey-corrected for 6 tests)

Fig.2B: Gene expression levels of $C x c / 1, C c / 20$ and $C c / 2$ in the skin

Cxcl1:

difference of the means IMQ running vs IMQ non-running 9.06 fold ( $95 \% \mathrm{Cl}: 2.92-28.18)$

interaction $\quad F(1,28)=9.174 \quad p=0.0052 \quad$ (two-way ANOVA)

IMQ running vs IMQ non-running $\quad \mathrm{p}=<0.0001 \quad$ (Tukey-corrected for 6 tests)

Ccl20:

difference of the means IMQ running vs IMQ non-running 4.62 fold $(95 \% \mathrm{Cl}: 1.2-17.78)$

interaction $F(1,28)=4.193 \quad p=0.0501 \quad$ (two-way ANOVA)

IMQ running vs IMQ non-running $\quad \mathrm{p}=0.0213 \quad$ (Tukey-corrected for 6 tests)

Fig.3A: Histological score of synovial hyperplasia and cellular infiltrate in the knee joints

Synovial hyperplasia:

difference of the means Ctrl vs IMQ 0.35 units $(95 \% \mathrm{Cl}: 0.10-0.60)$

main effect IMQ $\quad F(1,35)=8.146 \quad p=0.0072 \quad$ (two-way ANOVA)

Synovial cell infiltrate:

difference of the means Ctrl vs IMQ 0.54 units $(95 \% \mathrm{Cl}: 0.25-0.82)$

main effect IMQ $F(1,35)=14.81 \quad \mathrm{p}=0.0005 \quad$ (two-way ANOVA)

Fig.3B: Quantification of immunohistochemical detection of CD45 in knee synovium

difference of the means Ctrl non-running vs IMQ non-running 209.4 fold ( $95 \% \mathrm{Cl}$ : $105.0-418.6$ )

difference of the means Ctrl running vs IMQ running 12.39 fold $(95 \% \mathrm{Cl}: 6.21-24.75)$

interaction $\quad F(1,8)=85.74 \quad p<0.0001 \quad$ (two-way ANOVA)

Ctrl non-running vs IMQ non-running $\quad p<0.0001 \quad$ (Tukey-corrected for 6 tests) 
Fig.3C: Quantification of Immunohistochemical detection of F4/80 in knee synovium difference of the means Ctrl non-running vs Ctrl running 5.10 fold $(95 \% \mathrm{Cl}: 2.36$ - 11.01) difference of the means Ctrl running vs IMQ running 6.06 fold ( $95 \% \mathrm{Cl}: 2.81-13.07)$ interaction $F(1,8)=12.34 \quad p=0.0079 \quad$ (two-way ANOVA) Ctrl non-running vs Ctrl running $p=0.0006 \quad$ (Tukey-corrected for 6 tests) Ctrl running vs IMQ running $p=0.0003 \quad$ (Tukey-corrected for 6 tests)

Fig.4B: Gene expression levels of collagen 1a1 (Col1a1), collagen 3a1 (Col3a1) and transforming growth factor $\beta(T g f b)$ in the Achilles tendons

Col1a1:

difference of the means Ctrl vs IMQ 2.40 fold ( $95 \% \mathrm{Cl}: 1.11-5.29)$

main effect IMQ $\quad F(1,15)=5.788 \quad p=0.0295 \quad$ (two-way ANOVA)

\section{Col3a1:}

difference of the means 4.09 fold $(95 \% \mathrm{Cl}: 1.86-8.97)$

$\begin{array}{lll}\text { main effect IMQ } & F(1,15)=36.00 \quad p<0.0001 \quad \text { (two-way ANOVA) }\end{array}$

Tgfb:

difference of the means 2.08 fold $(95 \% \mathrm{Cl}: 1.47-2.95)$

main effect IMQ $F(1,15)=20.22 \quad p=0.0004 \quad$ (two-way ANOVA)

Fig.S1A: Gene expression levels of $C x c / 1, C c / 20$ and $C c / 2$ in colon

Cxcl1:

difference of the means acute DSS vs Ctrl 10.44 fold (95\%Cl: $4.18-16.69)$

main effect acute DSS $\quad F(1,26)=21.88 \quad p<0.0001 \quad$ (two-way ANOVA)

difference of the means chronic DSS vs Ctrl 8.050 fold ( $95 \% \mathrm{Cl}$ : $4.026-12.07$ )

main effect chronic DSS $\quad F(1,28)=54.54 \quad p<0.0001 \quad$ (two-way ANOVA) 


\section{CCl20:}

difference of the means acute DSS vs Ctrl 2.230 fold ( $95 \% \mathrm{Cl}: 0.4143-4.046)$

main effect acute DSS

$$
F(1,27)=10.58
$$

$p=0.0031$

(two-way ANOVA)

difference of the means chronic DSS vs Ctrl 1.594 fold (95\%Cl: $0.02243-3.165$ )

main effect chronic DSS

$F(1,27)=5.495$

$p=0.0267$

(two-way ANOVA)

Cc12:

difference of the means acute DSS vs Ctrl 6.699 fold (95\%Cl: $1.620-11.78)$

main effect acute DSS

$F(1,10)=24.46$

$p=0.0006$

(two-way ANOVA)

difference of the means chronic DSS vs Ctrl 1.983 fold (95\%Cl: $1.499-2.468)$

main effect chronic DSS

$F(1,28)=73.11$

$\mathrm{p}<0.0001$

(two-way ANOVA)

\section{Fig.S1B: Gene expression levels of $I d o 1, Z o-1$ and Reg3g in skin or colon}

Ido1:

difference of the means IMQ vs Ctrl 1.336 fold ( $95 \% \mathrm{Cl}: 0.5408-3.213$ )

main effect IMQ $\quad F(1,28)=4.914 \quad p=0.0349 \quad$ (two-way ANOVA)

difference of the means acute DSS vs Ctrl 17.35 fold (95\%Cl: $7.821-26.88)$

main effect acute DSS $\quad F(1,27)=44.30 \quad p<0.0001 \quad$ (two-way ANOVA)

difference of the means chronic DSS vs Ctrl 21.64 fold (95\% Cl: $14.05-29.23$ )

main effect chronic DSS $\quad F(1,28)=163.3 \quad p<0.0001 \quad$ (two-way ANOVA)

Zo-1:

difference of the means IMQ vs Ctrl 1.168 fold ( $95 \% \mathrm{Cl}: 0.4055-1.931)$

main effect IMQ $F(1,28)=11.20 \quad p=0.0023 \quad$ (two-way ANOVA)

Reg3g:

difference of the means acute DSS vs Ctrl 11.19 fold (95\%Cl: $4.183-18.19)$

main effect acute DSS $\quad F(1,27)=17.85 \quad p=0.0002 \quad$ (two-way ANOVA)

difference of the means chronic DSS vs Ctrl 57.38 fold (95\%Cl: $18.83-95.94)$

main effect chronic DSS $\quad F(1,28)=29.05 \quad p<0.0001 \quad$ (two-way ANOVA) 